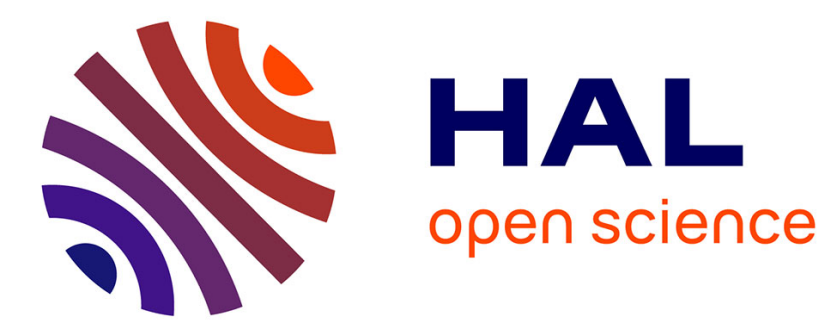

\title{
Les enjeux du développement durable
}

\author{
Alain Alcouffe, Sylvie Ferrari, Laurent Grimal
}

\section{To cite this version:}

Alain Alcouffe, Sylvie Ferrari, Laurent Grimal. Les enjeux du développement durable. Sciences de la Société, 2002, 52. hal-02116569

\section{HAL Id: hal-02116569 \\ https://hal.science/hal-02116569}

Submitted on 1 May 2019

HAL is a multi-disciplinary open access archive for the deposit and dissemination of scientific research documents, whether they are published or not. The documents may come from teaching and research institutions in France or abroad, or from public or private research centers.
L'archive ouverte pluridisciplinaire HAL, est destinée au dépôt et à la diffusion de documents scientifiques de niveau recherche, publiés ou non, émanant des établissements d'enseignement et de recherche français ou étrangers, des laboratoires publics ou privés. 


\title{
- Les enjeux du développement durable
}

\author{
A. Alcouffe, LIRHE, Université des Sciences Sociales de Toulouse \\ S. Ferrari, CERESUR, Université de La Réunion \\ L. Grimal, LIO, Université de Haute Alsace
}

S'il est vrai que les réflexions sur la relation entre les activités économiques et l'environnement étaient déjà présentes dans la philosophie antique, elles prennent une place grandissante dans la seconde moitié du XXe siècle avec l'apparition des pollutions, des déchets issus de modes de consommation gaspilleurs de ressources, la surexploitation des systèmes naturels, la consommation croissante d'énergies d'origine fossile dans un contexte de croissance exponentielle de la population mondiale. Le développement durable est un concept qui apparaît dans les années 1960 au moment même où l'idée d'une croissance illimitée commence à être remise en cause (Meadows, 1972). La croissance zéro est alors considérée comme une solution qui permet d'assurer le développement économique tout en garantissant la protection de l'environnement.

\section{Développement durable, environnement et société}

La genèse du concept peut être appréciée par trois dates clefs. En 1972, lors de la Conférence mondiale des Nations Unies sur l'environnement humain à Stockholm, la notion "d'écodéveloppement" est adoptée par la communauté internationale. Ce premier pas traduit la volonté d'ouvrir la voie vers un développement compatible avec les équilibres naturels et la protection de l'environnement. Dès lors, l'idée d'un développement guidé à la fois par des considérations économiques, sociales mais aussi écologiques va peu à peu infiltrer les grands programmes internationaux: création $\mathrm{du}$ Programme des Nations Unies pour l'Environnement en complément du Programme des Nations Unies pour le Développement.

Quelques années plus tard, c'est l'apparition d'un nouveau type de pollution qui oriente les réflexions vers la nécessité d'agir de manière solidaire au niveau de la biosphère. En effet, les pollutions globales telles que les pluies acides, l'effet de serre qu'accompagne notamment 1'accroissement continu du $\mathrm{CO}_{2}$ dans l'atmosphère, l'accumulation des déchets nucléaires... sont autant de facteurs qui révèlent à l'homo oeconomicus les limites de son action. En 1988, avec la publication du rapport Brundtland, "Our common future " (CMED, 1988), le concept de développement durable est proposé à partir d'une approche environnementale qui découle d'un double constat : la fragilité de la biosphère et de ces mécanismes régulateurs, et la fragilité du développement lui-même. Le développement durable est alors défini comme suit: " un développement qui répond aux besoins du présent sans compromettre la capacité des générations futures à répondre aux leurs "1. Cette définition

\footnotetext{
${ }^{1}$ Les définitions du développement durable sont très nombreuses. Dès 1989, J. Pezzey recensait trente-sept définitions présentes dans la littérature. D'après les travaux de F. Hatem, ces définitions peuvent se répartir selon qu'elles adoptent une vision écocentrée du développement ou une vision anthropocentrée. Dans le premier cas, la préservation de l'environnement est réalisée pour lui-même (il s'agit de protéger tous les êtres vivants) alors que, dans le second cas la préservation de l'environnement est justifiée par l'utilité que celui-ci procure à l'homme. Mais, parmi les partisans de cette deuxième vision selon laquelle le développement durable est celui qui permet d'assurer le maintien ou l'augmentation dans le temps du "bien être humain", les désaccords sont forts. La durabilité peut être très faible, faible ou forte (Faucheux \& Noël, 1995). Quoi qu'il en soit, cette opposition entre l'environnementalisme anthropocentrique et humaniste et le biocentrisme de la Deep Ecology relève d'un débat assez stérile (Bourg, 2001)
} 
est progressivement adoptée par la communauté scientifique. C'est également la définition la plus largement médiatisée. Le développement durable repose sur donc plusieurs principes : un principe de solidarité avec les générations futures et avec les populations présentes sur la planète, un principe de précaution et un principe de participation de tous les acteurs de la société civile au processus de décision. Il exige aussi de passer d'une approche réparatrice des dégâts environnementaux jusqu'alors dominante à une approche préventive.

La popularité de la définition proposée dans le rapport Brundtland peut s'expliquer par son imprécision et son caractère général. Elle permet en effet de masquer nombre de conflits potentiels ${ }^{2}$. D'une part, face à la multiplication tant des pollutions globales (effet de serre anthropogénique, déplétion de la couche d'ozone, etc.) que des micro pollutions l'objectif d'équité intergénérationnelle exige pour le moins de maintenir l'intégrité de la biosphère et de ses mécanismes régulateurs. Il s'agit donc d'introduire une responsabilité éthique vis à vis de nos descendants en faisant implicitement référence à des stratégies de préservation du patrimoine naturel et des écosystèmes. D'autre part, la définition fait référence à un objectif d'équité intragénérationnelle. Cet objectif implique à la fois une responsabilité éthique des pays développés vis à vis des pays moins développés mais également au sein des pays développés des liens de solidarité accrus entre les personnes socialement intégrées et les personnes exclues des systèmes économiques et sociaux. En somme, comme la définition du développement durable vise à nommer deux défis plutôt qu'à ordonner les actions à entreprendre, elle génère autour d'elle un consensus. Néanmoins, ce consensus vole en éclats dès qu'il s'agit de dématérialiser et "décarboniser" les procédés de consommation et de production, ou encore de modifier la répartition des richesses entre les différents pays et au sein de chaque pays.

La troisième date clé est 1992, année où se déroule à Rio de Janeiro la deuxième conférence mondiale des Nations Unies sur l'environnement.À ce "Sommet de la Terre ", les réflexions portent la marque de changements majeurs au niveau mondial : depuis la conférence de Stockholm, la population mondiale est passée de 3,5 milliards d'individus à 5 milliards, l'appauvrissement des pays du Sud s'est accentué ; le remboursement de la dette absorbe l'aide publique du Nord, etc. La conférence de Rio aboutit sur plusieurs points : rédaction des principes de la "Déclaration de Rio", d'un programme d'actions pour le XXIe siècle avec l'Agenda 21 et de deux conventions: convention cadre sur le changement climatique et convention sur la diversité biologique.

Parmi les principes qui sous-tendent le développement durable, nous pouvons mentionner: la précaution en matière d'environnement, l'intégration de la protection de l'environnement comme partie intégrante du processus de développement, la responsabilité et la solidarité internationale, le principe du pollueur-payeur, la participation pour une nouvelle forme de gouvernance. La mise en œuvre de ces différents principes doit permettre d'atteindre les objectifs de lutte contre la pauvreté, de préservation des équilibres de la biosphère et des ressources environnementales dans une perspective de long terme, de favoriser les modes de production et de consommation durables i.e. dotés d'une efficacité énergétique élevée, tout cela dans le respect des générations présentes et futures.

Cependant, malgré cette prise de conscience des problèmes environnementaux et de sous-développement, malgré l'accord sur les principes d'une plus grande défense du patrimoine naturel alliée à une amélioration de la qualité de la vie pour l'ensemble de l'humanité, les actes qui traduiraient de manière conséquente ces principes sont encore trop

\footnotetext{
${ }^{2}$ Pour certains auteurs, tels que S. Latouche (1994), l'imprécision de la notion de développement durable en fait un "concept alibi". Ce dernier permet d'aménager à la marge les modes de développement actuels en intégrant quelques paramètres environnementaux.
} 
peu nombreux. Et, lorsqu'ils existent, ils relèvent plus d'actes isolés que d'une stratégie politique à grande échelle.

Dès lors, il n'est pas surprenant que, dix années plus tard, divers indicateurs de développement durable montrent combien la pression exercée par l'activité humaine sur l'environnement est sérieuse et croissante, et combien les inégalités n'ont cessé de progresser. Examinons succinctement certains de ces indicateurs.

\section{- Changement climatique :}

Le réchauffement climatique, très probablement induit par l'augmentation des gaz à effet de serre et dont la responsabilité incombe pour l'essentiel aux émissions de gaz carbonique, pourrait avoir de fortes conséquences. Si l'évolution actuelle se prolonge les variations de température ${ }^{3}$ auront des effets conséquents sur les écosystèmes, la répartition des populations et les conditions sanitaires notamment en raison d'un fort accroissement des irrégularités temporelles et géographiques des précipitations. On ne peut non plus exclure des chocs de grande ampleur notamment sur la circulation océanique (IPCC, 2002).

\section{- Biodiversité :}

En l'absence d'une préservation suffisante, la diversité biologique est menacée d'appauvrissement de manière chronique. Selon les Nations Unis $(2001$, a) qui se réfèrent à la liste rouge 2000 des espèces menacées publiée par l'Union internationale pour la conservation de la nature et de ses ressources - Alliance mondiale pour la nature (UICN), la troisième phase d'une crise mondiale majeure de la disparition des espèces s'amorce. La liste rouge propose un jeu de critères quantitatifs pour l'évaluation de la situation des espèces et de leur habitat. En 2000, l'UICN met en avant le fait que de nombreuses espèces pourraient disparaître au cours des premières décennies du XXIe siècle si la communauté mondiale n'accentue pas considérablement son appui, sa participation et son engagement en faveur de la préservation des espèces. La liste rouge comprend notamment 11046 espèces menacées de disparition et 816 espèces qui ont déjà disparu ou ont disparu à l'état sauvage. Ces 11046 espèces menacées de disparition, bien que représentant moins de $1 \%$ des espèces connues au monde, représentent $24 \%$ de toutes les espèces de mammifères et $12 \%$ de toutes les espèces d'oiseaux.

\section{- Pauvreté :}

Toujours selon les Nations Unis (2001, b), entre 1990 et 1998, le taux de pauvreté dans les pays en développement, établi sur la base d'un seuil mondial de pauvreté fixé à 1 dollar par jour, a diminué de 29 à $23 \%$. Si cette tendance décroissante pouvait être maintenue à ce rythme, le taux de pauvreté serait en 2015 quasiment inférieur de moitié à celui de 1990. Mais en raison de la croissance démographique, le nombre total de personnes vivant dans la pauvreté a seulement légèrement diminué au cours des années 90, passant de 1,3 à 1,2 milliard de personnes. De plus, cette évolution globale cache de fortes disparités régionales. Par exemple, en Afrique subsaharienne, le taux de pauvreté est demeuré largement inchangé entre 1990 et 1998, se situant autour de $48 \%$, et le nombre de personnes vivant dans la pauvreté est passé de 217 à 291 millions.

Par ailleurs, les inégalités dans la répartition des revenus à l'échelle mondiale se sont accentuées au cours des dernières décennies, en raison essentiellement de l'écart qui se creuse

\footnotetext{
${ }^{3}$ Le Groupe d'experts intergouvernemental sur l'évolution du Climat (IPCC, 2001) a publié en 2001 son troisième rapport d'évaluation. Selon ce rapport, le réchauffement serait plus important que prévu, avec une augmentation de la température moyenne globale de $1,4^{\circ} \mathrm{C}$ à $5,8^{\circ} \mathrm{C}$ d'ici la fin du siècle. Les auteurs du rapport affirment que la majeure partie du réchauffement climatique des 50 dernières années est due à la combustion des énergies fossiles. Pour éviter que le réchauffement de la planète ne produise ses effets les plus extrêmes, on estime que les émissions de dioxyde de carbone devront être réduites de moitié d'ici 2050.
} 
entre les pays les plus nantis et les pays les plus défavorisés. Le rapport entre le revenu moyen des $20 \%$ les plus riches du monde et le revenu moyen des $20 \%$ les plus pauvres qui était de 60 à 1 en 1991, a atteint 78 à 1 en 1994. Les $20 \%$ les plus pauvres de la planète ne reçoivent que $1,1 \%$ du revenu mondial, ce qui représente une baisse par rapport à 1991, année où ils recevaient $1,4 \%$ du revenu mondial.

\section{- Évolution des modes de production et de consommation :}

L'un des principaux enjeux du développement durable réside dans la capacité d'une économie à générer une croissance de long terme, pérenne. La création de richesses est en effet une condition nécessaire du développement et du progrès économique et social. C'est du moins le postulat sous-jacent à une définition anthropocentrée du développement durable. Mais cette croissance doit prendre en compte le maintien des stocks de capitaux et la bonne gestion du patrimoine naturel. Il s'agit donc d'améliorer la productivité globale de l'économie à travers la dématérialisation des activités et la limitation des consommations d'énergie, de matières premières et d'espace. Dans cette perspective, les indicateurs doivent apporter des éléments de réponse sur l'éventuel couplage ou découplage de la croissance économique au regard des différentes catégories de capitaux ou de ressources (Spangenberg, 2000). Il s'avère que ce découplage entre la croissance économique et les pressions exercées sur l'environnement n'est dans le meilleur des cas que partiel. Ainsi, pour un pays comme la France, le dernier Rapport sur l'état de l'environnement de l'Institut Français de l'Environnement pour la période 19982001 (IFEN, 2002), indique que la pression sur les territoires, les milieux et les écosystèmes se sont significativement accrues durant ces trois années. Il s'avère que les quantités de déchets et les émissions de gaz carbonique continuent de croître au même rythme que la consommation.

En définitive, depuis le sommet de Rio et la médiatisation croissante de la notion de développement durable, l'environnement a continué à se dégrader et la pauvreté, au niveau mondial, n'a finalement que très peu reculé. Bjorn Lomborg, un ancien militant de Greenpeace en est venu à mettre en question ces conclusions des environnementalistes. Dans sa critique systématique des discours sur l'environnement, Bjorn Lomborg conteste la réalité des périls dénoncés par les environnementalistes, que ce soit la surpopulation, les pesticides, l'effet de serre, l'épuisement des ressources fossiles, l'eau, etc. Cette critique a un aspect certainement salutaire : le souci de l'environnement peut aisément verser dans le millénarisme et les prévisions alarmantes ont certainement besoin d'être contrôlées et rigoureusement évaluées mais, en même temps, en lisant ces critiques, on ne peut s'empêcher de penser aux problèmes traditionnels de la méthodologie des sciences. Celle-ci a bien mis en évidence qu'il est impossible de prouver qu'une proposition est "vraie" tandis que l'on peut plus facilement prouver qu'une proposition est "fausse". Bjorn Lomborg, fort de ses compétences en statistique, s'en donne à cœur joie de démonter les "preuves" avancées par les environnementalistes. Mais, en dénonçant les erreurs des environnementalistes, Lomborg a lui-même tendance à passer de la réfutation (non, tout ne va pas aussi mal que le prétendent que les environnementalistes) à l'affirmation -elle même impossible à démontrer - que tout va mieux qu'hier et moins bien que demain. Par ailleurs, le courant environnementaliste n'est peut-être pas pour rien dans les raisons de l'optimisme de Lomborg -ainsi dans le cas du trou d'ozone, n'est ce pas la mise en évidence du phénomène et le rôle qu'y jouaient certains gaz qui a provoqué une limitation de leur usage et l'amélioration possible de la situation ? Les économistes ont mis en évidence l'existence de prophéties auto-réalisatrices, peut être faut-il porter au crédit de ceux qui ont fait connaître des prévisions alarmantes par exemple en matière de population que les hypothèses hautes ne semblent "heureusement" pas se réaliser. Hegel remarquait que "l'oiseau de Minerve -(la philosophie, la science) --ne prend son vol qu'au crépuscule". Ces remarques soulignent que ce n'est pas dévaloriser la "science" que de conclure que les décisions en matière d'environnement ne peuvent être remises entre les mains 
des seuls experts. C'est d'ailleurs là une des explications de l'échec du "contrat" signé à Rio qui n'a été respecté sur aucun de ses deux volets. Parmi les raisons de cet échec, il y a très certainement la complexité de définir des politiques environnementales et sociales cohérentes, de les faire accepter et appliquer par les populations et les milieux industriels, mais aussi de construire un nouveau lien institutionnel entre tous les acteurs du développement durable. En effet, le développement durable repose sur la participation de l'ensemble des acteurs de la société civile; le processus décisionnel change de dimension.

\section{Environnement et gouvernance}

La question de la forme de gouvernance pour un développement durable se pose donc avec une forte acuité. La gouvernance est un concept à l'origine économique qui apparaît dans les travaux de Coase en 1937. Il désigne alors l'ensemble des dispositifs de coordination interne à une entreprise qui lui permettent d'organiser efficacement certains échanges : c'est le gouvernement d'entreprise ou corporate governance.

En général, la gouvernance désigne l'ensemble des relations de pouvoir au sein d'une société ainsi que les modes d'arbitrage entre les différents détenteurs du pouvoir. Le gouvernement désigne donc l'exercice du pouvoir politique sur une communauté d'individus et, par extension, le pouvoir qui dirige un Etat. Le gouvernement n'est donc qu'un des acteurs de la gouvernance. Si la question de la gestion des affaires publiques se déplace aujourd'hui du gouvernement vers la gouvernance, c'est parce que les gouvernements sont de plus en plus contraints dans leur action par l'influence d'autres acteurs (Etats étrangers, organisations supranationales comme l'Union européenne, acteurs privés, milieux associatifs, institutions, etc.). Ils ne peuvent pas définir et appliquer seules les règles qui conduisent les individus et les groupes sociaux. La gouvernance désigne précisément les mécanismes d'élaboration et de contrôle de ces règles (Lerin, 1997).

La gouvernance au service du développement durable a donc un contenu original. En imposant une transformation des formes de la décision et de l'action publique ainsi que des institutions, elle contient une éthique des comportements (lutte contre la corruption ou contre la délinquance écologique, transparence), de la précaution dans les actions à entreprendre, et favorise les partenariats garant de l'intégration des dimensions sociales et environnementales. Concrètement, c'est un processus décisionnel continu et coopératif entre des acteurs dotés d'intérêts conflictuels.

Un exemple de gouvernance appliquée au développement durable est celui lié aux négociations internationales autour du changement climatique. À la suite de la convention sur le changement climatique signé en 1992 à Rio, les Etats les plus riches se sont engagés à ramener leur émission de gaz à effet de serre à leur niveau de 1990 d'ici à l'an 2000. En décembre 1997, cette convention a été complétée par le protocole de Kyoto. Ce protocole de Kyoto, plus contraignant, impose aux pays industrialisés de réduire, d'ici 2012 leurs émissions d'au moins $5 \%$ par rapport à leur niveau de 1990. Ce n'est qu'en novembre 2001, à Marrakech, que les pays signataires du protocole sont parvenus à un accord sur les modalités d'application qui permet de le rendre enfin opérationnel. Par conséquent, le choix et la mise en œuvre effective des politiques et instruments (mise en œuvre conjointe, ventes de droits d'émission, mécanismes de "développement propre", etc.) relèvent essentiellement d'un processus politique et fait l'objet de négociations entre l'ensemble des acteurs socioéconomiques. Une validation de ce choix par les différents acteurs, sous la forme d'un consensus ou d'un arbitrage conflictuel entre eux, s'avère nécessaire pour assurer sa légitimité et éviter les conflits concernant son interprétation et son utilisation. Par ailleurs, les politiques, mesures et instruments qui seront retenus lors des différentes phases du processus 
de négociation sont par nature évolutifs. La pertinence d'une négociation est donc établie dans le contexte des projets et des préoccupations d'une société ; voire dans le contexte d'un projet de société.

Il s'avère donc qu'une "bonne gouvernance" est indispensable pour un développement durable. L'exercice n'est pas facile car il suppose de trouver les " justes" niveaux d'expression, de diversifier les formes de décision et d'encourager la démocratie participative, de délivrer une information transparente globale et solide, de constituer un dialogue libre et constructif, de multiplier les partenariats, de favoriser la transversalité, de croiser les logiques et pratiques sectorielles, de développer des procédures d'évaluation continue, collectives et contradictoires... Le Programme des Nations Unies pour le Développement donne la définition suivante : "La gouvernance peut être considérée comme l'exercice des pouvoirs économique, politique et administratif pour gérer les affaires des pays à tous les niveaux. Elle comprend les mécanismes, procédés et institutions [...]. La bonne gouvernance est [...] participative, transparente et responsable. Elle est aussi efficace et équitable. La bonne gouvernance assure que les priorités politiques, sociales et économiques sont fondées sur un large consensus dans la société et que les voix des plus pauvres et des plus vulnérables sont au cœur du processus de décision sur l'allocation des ressources pour le développement" (PNUD, 1997).

La mise en place d'une “ bonne gouvernance " est d'autant plus nécessaire que l'État, en particulier dans les pays industrialisés, après avoir reçu implicitement de la communauté qu'il représente un mandat pour protéger l'environnement, semble déléguer à son tour à l'entreprise et-ou aux Organisations Non Gouvernementales la charge de la protection de l'environnement. Cette volonté de fonder un développement durable sur le volontariat des entreprises plutôt que sur des politiques environnementales publiques est une tendance lourde. Par exemple, dans le cadre de la préparation du Sommet mondial pour le développement durable qui a eu lieu à Johannesburg du 26 août au 4 septembre 2002 les Nations Unies, dans les documents préparatoires, ont mis l'accent sur les alliances entre le secteur public et le secteur privé. En effet, celles-ci constitueraient un vecteur puissant de création de capacités, de transferts de technologie et une passerelle entre l'aide publique au développement et les investissements étrangers directs. En effet, en présence d'incertitudes fortes dans l'avenir sur les conventions environnementales, un élément clé de la coordination économique est l'intervention de partenariats collectifs pour offrir des connaissances et visions du monde communes et de nouvelles normes de comportement ayant un effet de légitimation d'une orientation ou d'une préoccupation donnée.

Dans ce contexte, la définition d'une "bonne gouvernance" pour un développement durable est d'autant plus importante qu'il est difficile de faire l'hypothèse que les évolutions dans les comportements publics, les régulations environnementales ou les pratiques stratégiques des firmes satisferont automatiquement aux conditions de durabilité économique, sociale et écologique. Par exemple, de nombreux débats à propos de la pertinence des politiques de développement durable concernent les potentialités du changement technologique à réduire les émissions polluantes et à susciter un usage efficient des ressources naturelles. La contribution du changement technologique à la diminution de l'intensité environnementale des produits, des processus de production et des services est primordiale même si tout progrès réalisé dans le domaine de l'éco-efficacité peut être compensé par un effet de rebond (Binswanger, 2001). Or, les grands groupes industriels sont en position d'influencer la direction de l'innovation technologique environnementale. De même, s'il est courant d'avancer le principe de précaution pour les décisions comportant des risques environnementaux, la contrepartie de son application s'apparente souvent à un gel quasiirréversible des positions concurrentielles en matière technologique acquises au moment de la décision. Cette situation peut favoriser des comportements stratégiques de la part des 
différents acteurs, en l'occurrence des firmes, afin d'orienter la décision finale dans le sens de leur intérêt. Les nouvelles formes de gouvernance doivent alors garantir la prise en compte de responsabilités sociales plus larges dans la recherche de stratégies de type "win-win" (Faucheux S., Nicolaï, 1998).

En somme, les réflexions sur les modes de gouvernance au service d'un développement durable ont induit de nouvelles méthodologies inhérentes à l'élaboration des normes, des réglementations, des mesures économiques et d'aide à la décision. Elles conduisent à mettre en œuvre des systèmes d'apprentissage collectif basés sur des processus consultatifs et participatifs. L'interaction au sein de ces dispositifs engendre ainsi une légitimité des mesures environnementales retenues. Par exemple, le récent développement de certains instruments de protection de l'environnement tels que les engagements volontaires ou les écolabélisations souligne l'évolution du cadre institutionnel des politiques environnementales. Cette tendance croissante à réunir des parties prenantes hétérogènes revient ainsi à réaffirmer les principes du développement durable.

\section{Développement durable, culture et gouvernance}

Le renouvellement des ressources a une dimension culturelle dès lors que l'on définit assez largement le domaine de la culture. Les ressources culturelles incluent par exemple les sites, les paysages, les monuments, les objets et les documents associés aux activités humaines présentes et passées. Elles ont aussi un aspect immatériel comme les mythes, les idées, les normes sociales, la langue que chaque génération reçoit, enrichit et transmet. La plupart des ressources culturelles sont uniques et non renouvelables. Elles sont apparues ou ont été délibérément créées dans des lieux particuliers à des dates déterminées par des individus donnés. La durabilité relative à l'environnement et la préservation des ressources culturelles sont donc largement complémentaires: les valeurs culturelles et l'histoire des populations représentent des adaptations ou des réponses à l'environnement. La préservation de la biodiversité et la préservation des langues moins diffusées sont deux versants d'une même protection des dangers qui pourraient naître d'une réduction des espèces et d'une uniformisation des modes de vie et des langages. C'est là un souci qui anime les défenseurs des "peuples premiers" et que partagent tous ceux qui se préoccupent des dimensions spatiale et culturelle du développement durable ${ }^{4}$.

Aux côtés de la culture et de ses déclinaisons matérielles et spirituelles, l'espace constitue certainement une dimension fondamentale des processus orientant le développement durable. Le choix des outils d'aides à la décision et des moyens à mettre en œuvre peuvent changer selon l'entité spatiale considérée, Les dimensions locale, régionale ou nationale du développement contiennent différentes facettes de la préservation de la qualité de l'environnement, de la nécessité de diminuer la production de déchets ou encore des modalités de gestion des ressources naturelles multi-fonctions. Sur ce point, l'eau est un exemple de ressource qui, du fait de cette particularité, nécessite une approche spatialisée. Sa multi-fonctionnalité se décline à travers différents services, marchands ou non, qu'elle procure: services d'épuration et de recyclage (dilution, auto-épuration...), services en énergie motrice, services écologiques, services d'alimentation en eau potable, services d'alimentation hydrique de la végétation, services récréatifs. Dans le cas de la France, par exemple, le législateur a introduit des outils de gestion et de

\footnotetext{
${ }^{4}$ L'Eurocongrès 2000 des espaces occitan et catalan, suite de manifestations destinées à rappeler et promouvoir une histoire partagée millénaire à cheval sur deux -Etats nations, leur a fait une large place. C'est ainsi qu'il a organisé le colloque éponyme du présent numéro de Sciences de la Société les 25 et 26 octobre 2002.
} 
planification de l'eau adaptés à des niveaux d'approche distincts: création des SDAGE (Schéma Directeur d'Aménagement et de Gestion des Eaux) au niveau des bassins hydrographiques (niveau global) et des SAGE (Schéma d'Aménagement et de Gestion des Eaux) au niveau des sous-bassin (niveau local). Dans le cadre d'un SAGE, les acteurs impliqués dans sa protection et sa gestion relèvent des collectivités territoriales, des services de l'Etat, et des usagers de l'eau dans le cadre d'une commission locale de l'eau.

Par conséquent, avec la loi sur l'eau du 3 Janvier 1992 (modifiée) ${ }^{5}$, le cadre spatial et temporel considéré traduit une approche intégrée de la ressource où les milieux naturels (écosystèmes) et leur fonctionnement sont pris en compte dans la gestion de l'eau (bassins hydrographique, cycle de l'eau). La ressource fait alors l'objet d'une approche patrimoniale au centre de laquelle figurent la préservation des écosystèmes, la protection contre les pollutions, la restauration de la qualité, sa valorisation économique ainsi que sa répartition entre différents usages concurrents (Ferrari, Point, 2002).

Dans ce contexte, les formes de gouvernance guidant le développement durable sont nécessairement liées à l'histoire des populations concernées, à leurs pratiques culturelles et à l'espace qu'elles occupent à un moment donné. Alors que le Sommet de Johannesburg présente un bilan en demi-teinte des efforts déployés depuis celui de Rio, la nécessité de poursuivre la réflexion sur la gouvernance est donc plus que jamais d'actualité. Le présent numéro de Sciences de la Société a précisément pour objet d'étudier différents problèmes d'ordre institutionnels ayant trait aux formes de gouvernance adaptées au développement durable. Il s'intéresse notamment aux modalités participatives impliquant les acteurs dans les politiques de développement durable appliquées à l'échelle d'une région (variété des exploitations agricoles et développement rural, rôle du territoire rural dans la dynamique de développement), d'un secteur d'activité (rôle de la multifonctionnalité de l'agriculture notamment) ou encore d'une ressource naturelle (l'eau).

\section{Références :}

Binswanger M.(2001), "Technological progress and sustainable development: What about the rebound effect?", Ecological Economics, 36 (1) : 119 - 132.

Bourg D. (2001), "le nouvel âge de l'écologie", Le Débat, n¹13, pp. 92-109.

Commission Mondiale sur l'Environnement et le Développement (1988), Notre avenir à tous, traduction de Brundtland G.H. (1988), Our Common Future, Ed 7 du Fleuve, Montréal.

Faucheux S., Nicolaï I. (1998), "les firmes face au développement soutenable : changement technologique et gouvernance au sein de la dynamique industrielle", Revue d'Economie Industrielle, 83 (1) : 127-146.

Faucheux S., Noël J-F. (1995), L'économie de l'environnement et des ressources naturelles, Edition Armand Colin.

Ferrari S., Point P., (Eds), (2002), Préservation et valorisation de la ressource en eau. Application au domaine littoral, Edition Karthala, à paraître.

Hatem F. (1990), "Le concept de développement soutenable : une origine récente, une notion ambiguë, des applications prometteuses", Economie Prospective Internationale, 44 (4) : 101 $-117$.

\footnotetext{
${ }^{5}$ Selon l'article 1 de la loi : "L'eau fait partie du patrimoine commun de la nation. Sa protection, sa mise en valeur et le développement de la ressource utilisable, dans le respect des équilibres naturels, sont d'intérêt général. L'usage de l'eau appartient à tous dans le cadre des lois et règlements ainsi que des droits antérieurement établis".
} 
IPPC (2001) à ajouter.

IPCC (2002), Climate Change 2001: Synthesis Report, Third Assessment Report of the Intergovernmental Panel on Climate Change, Watson R.T. (Eds.), Cambridge University Press.

Ifen (2002) à ajouter.

Bjorn Lomborg, (2001), The Skeptical Environmentalist : Measuring the Real State of the World, Cambridge University Press, Cambridge.

Latouche S. (1994), "Développement durable : un concept alibi. Main invisible et mainmise sur la nature", Revue Tiers Monde, XXXIII (137) : 77-94.

Lerin F. (1997), "La gouvernance : relations de pouvoir et modes d'arbitrage", Courrier de la Planète, Réforme de l'État et nouvelle gouvernance, $\mathrm{n}^{\circ} 41$.

Meadows D.H., Meadows D.L., Randers J., Behrens W.W. (1972), The limits of growth: A report for the Club of Rome's project on the predicament of mankind, Earth Island Press, London.

Nations Unis (2001, a), Situation mondiale de la diversité biologique, Rapport du Secrétaire général Commission du développement durable constituée en comité préparatoire du Sommet mondial pour le développement durable, E/CN.17/2001/PC/18, Session d'organisation 30 avril-2 mai 2001

Nations Unis (2001, b), Lutte contre la pauvreté, Rapport du Secrétaire général, Commission du développement durable constituée en comité préparatoire du Sommet mondial pour le développement durable, E/CN.17/2001/PC/5, 30 avril-2 mai 2001

Pezzey J. (1989), Economic analysis of sustainable growth and sustainable development, World Bank, Environment Department, Working Paper $\mathrm{n}^{\circ} 15$.

PNUD (1997), La Gouvernance en faveur du développement humain durable, Document de politique générale du PNUD, PNUD New York.

Spangenberg J. (2000), "The environmental Kuznets curve : A methodological artefact?", Transitions towards a sustainable Europe - Ecology - Economy - Policy, 3rd Biennial Conference of ESEE, University of Vienna, 3-6 May. 